\title{
Mental model-based repeated multifaceted (MRM) intervention design: a conceptual framework for improving preventive health behaviors and outcomes
}

\author{
Mazbahul G. Ahamad ${ }^{1 *}$ (D) and Fahian Tanin ${ }^{2}$ (D)
}

\begin{abstract}
Objective: Field interventions employed to improve preventive health behaviors and outcomes generally use wellestablished approaches; however, recent studies have reported that health education and promotional interventions have little to no impact on health behaviors, especially in low- and middle-income countries. We aimed to develop a conceptual framework to improve intervention designs that would internalize these concerns and limitations.

Results: We identified three major experimental design- and implementation-related concerns associated with mental models, including the balance between the treatment and control groups, the treatment group's willingness to adopt suggested behaviors, and the type, length, frequency, intensity, and sequence of treatments. To minimize the influence of these aspects of an experimental design, we proposed a mental model-based repeated multifaceted (MRM) intervention design framework, which represents a supportive intervention design for the improvement of health education and promotional programs. The framework offers a step-by-step method that can be used for experimental and treatment design and outcome analysis, and that addresses potential implementation challenges.
\end{abstract}

Keywords: Balance test, Experimental design, Health education and promotion, Impact evaluation of health policy and social program, Intervention design, Mental model mapping, Monetary and behavioral interventions, Multifaceted intervention, Preventive health behavior and outcome, Water, Sanitation, Hygiene

\section{Introduction}

Public health intervention research generally applies existing, well-known experimental designs to improve preventive health behaviors and outcomes; however, recent literature has reported that water, sanitation, and hygiene (WASH)-related health education and promotional interventions have had little to no effect on these behaviors and outcomes [1-4]. These types of interventions may be minimally effective or ineffective due to

\footnotetext{
*Correspondence: mahamad2@unl.edu; mg.ahamad@gmail.com 1 University of Nebraska-Lincoln, 140 Keim Hall, 1825 N 38th St, Lincoln, NE 68583, USA
}

Full list of author information is available at the end of the article inappropriate (or appropriate but ineffective) experimental and treatment designs, the influence of contextual factors [5], or unobserved events, especially in low- and middle-income countries $[1,2,4,6]$. Intervention programs typically require a change in behavior among the members of a treatment group (e.g., individuals or households); this change is intended to improve preventive health behaviors and outcomes [7]. However, behavioral changes are directly associated with various factors, for example, the experimental group's willingness to accept the suggested behaviors, their cognitive ability to adapt to new behaviors, and their mentality (or mental model) [811]. Mentality is particularly important because "mental models are how we understand the world. Not only do

(c) The Author(s) 2021. This article is licensed under a Creative Commons Attribution 4.0 International License, which permits use, sharing, adaptation, distribution and reproduction in any medium or format, as long as you give appropriate credit to the original author(s) and the source, provide a link to the Creative Commons licence, and indicate if changes were made. The images or other third party material in this article are included in the article's Creative Commons licence, unless indicated otherwise in a credit line to the material. If material is not included in the article's Creative Commons licence and your intended use is not permitted by statutory regulation or exceeds the permitted use, you will need to obtain permission directly from the copyright holder. To view a copy of this licence, visit http://creativeco mmons.org/licenses/by/4.0/. The Creative Commons Public Domain Dedication waiver (http://creativecommons.org/publicdomain/ zero/1.0/) applies to the data made available in this article, unless otherwise stated in a credit line to the data. 
they shape what we think and how we understand but they shape the connections and opportunities that we see." [12]. Similarly, "mental models are how we simplify complexity, why we consider some things more relevant than others, and how we reason" [12]. These factors can create major challenges to intervention design and during the implementation phases of public health research and program development. Increasing the effectiveness of interventions that focus on improving preventive health behaviors may be difficult [13] due to the complex nature and context-dependency of these behaviors $[5,14$, 15].

When we reviewed interventions that focus on preventive health behaviors, we observed three common concerns among interventions that reported little to no effect on behavioral outcomes. First, these intervention approaches implicitly assumed that all treated individuals or households were identical, with similar cognitive capacities or mental models; such an assumption increases the likelihood of sample imbalance and bias in final outcome estimates [16]. The standard sample balance tests disregard behavioral or cognitive factors [17], despite frequently taking into consideration more easily observable socioeconomic and demographic characteristics (e.g., income, wealth, age) and, occasionally, knowledge level of health behaviors. Second, behavioral change programs require long-term interventions and frequent follow-ups [18], and treatment effects often wane over time, particularly after single-treatment-based interventions [19]. Third, the treatment groups' exposure to the intervention does not vary or repeat throughout the study period, which is not comparable to the changing conditions in the real world [20].

Although some corrective measures can be applied to address the treatment-waning effect (e.g., as occurs in repeated or multifaceted interventions), no assessments that could be used to understand a treatment group's mental model as associated with a public health intervention design are currently available. From a public health perspective, an individual's mental model explains the experimental subject's cognitive ability to perceive potential health risks and perform the necessary decision-making that influences health outcomes. Therefore, identifying and understanding the mental models of both the treatment and control groups is important to the design of cognitive ability- or mental model-based treatments that can be used to generate the expected intervention effects in field experiments.

The three confounding concerns that we identified can, in combination, represent significant interventionrelated sources of poor health behaviors and outcomes. A potential experimental design framework aimed to improve preventive health behavior intervention designs in response to low or no intervention effects could consider the concerns associated with mental model-based balance tests, the waning of the treatment effect, and the persistence of intervention impacts. In the study, we described the development of a conceptual framework for a mental model-based repeated multifaceted (MRM) intervention design intended to improve intervention impacts by internalizing these concerns and limitations when the likely outcomes of preventive health behaviors improvement programs are assessed.

\section{Main text \\ Materials and methods Identification of key limitations and concerns}

In stage one, we identified limitations and concerns regarding intervention designs and effects. First, we conducted a narrative review of published systematic reviews to identify the structure and common components of WASH-related preventive health education and promotion interventions and outcomes. Second, we reviewed field experiment-based empirical literature regarding the limitations and concerns indicated for both statistically significant and insignificant low or no intervention effects. We only considered peer reviewed systematic reviews and articles based on field experiments in resource-poor low- and middle-income countries. We used our search strategy with PubMed and Cochrane Library to source peer-reviewed articles published from January 2010 to June 2020 that used experimental field data (Additional file 1: Table S1). Finally, 86 systematic reviews and 49 empirical articles from PubMed and 129 trials from Cochrane Library were identified as useful in exploring the "common limitations" of existing experimental design approaches and intervention outcomes that were specifically mentioned in either the results and discussion or the study limitation sections, or both.

\section{Framework development}

Our proposed MRM intervention design framework is based on the major concerns cited in existing systematic and scoping reviews, trials, and recent empirical studies regarding design approaches, treatment frequency and components, sample balance variables, and the time dimensions of treatment interventions in published WASH-related field experiments (Table 1). At this stage, we searched the interdisciplinary literature (e.g., development economics, natural resources, behavioral economics) regarding the same concerns and limitations for interventions in public health. We then considered the different experimental approaches used in interdisciplinary fields to develop a modified intervention design that could internalize the common limitations (Fig. 1 following Additional file 2: Figure S1). 
Table 1 Major features and concerns of preventive health behavior-related interventions

\begin{tabular}{llll}
\hline Issues & Category & Key feature & Concern/advantage \\
\hline Approach & Single & Only one treatment & Treatment effect wanes over time \\
Frequency & Multifaceted & Multiple treatments & Persistent treatment effect \\
& Single intervention & One round & Treatment effect wanes over time \\
Balance test & Multiple intervention & Several rounds & Creates more persistent effect \\
& Socioeconomic & Income, wealth, age, sex, education & Mental model or cognitive capacity-related factors are mostly \\
& and demographic & & absent \\
Treatment component & Informational & Information-based letter & Less effective \\
& Educational & Education & Effective but depends on the curriculum \\
& Training & Hands-on experience & Effective but depends on the type and length of training \\
& Financial & In cash only & Attractive but ineffective if stopped \\
& Promotional & In kind or service & Highly effective with other treatments \\
& Behavioral & Weak or strong norm-based nudging & Highly effective with other financial treatments \\
& Mixed & Both financial and behavioral & More effective than either financial or behavioral alone \\
& Short-term & Less than one year & Treatment effects wanes over time \\
& Medium-term & One to five years long & Better than short-term intervention \\
& Long-term & More than five years & Creates a more persistent effect
\end{tabular}

We reviewed water, sanitation, and hygiene (WASH)-related prevention health education and promotional interventions, (e.g., systematic reviews, empirical evidence) to explore the major features and concerns

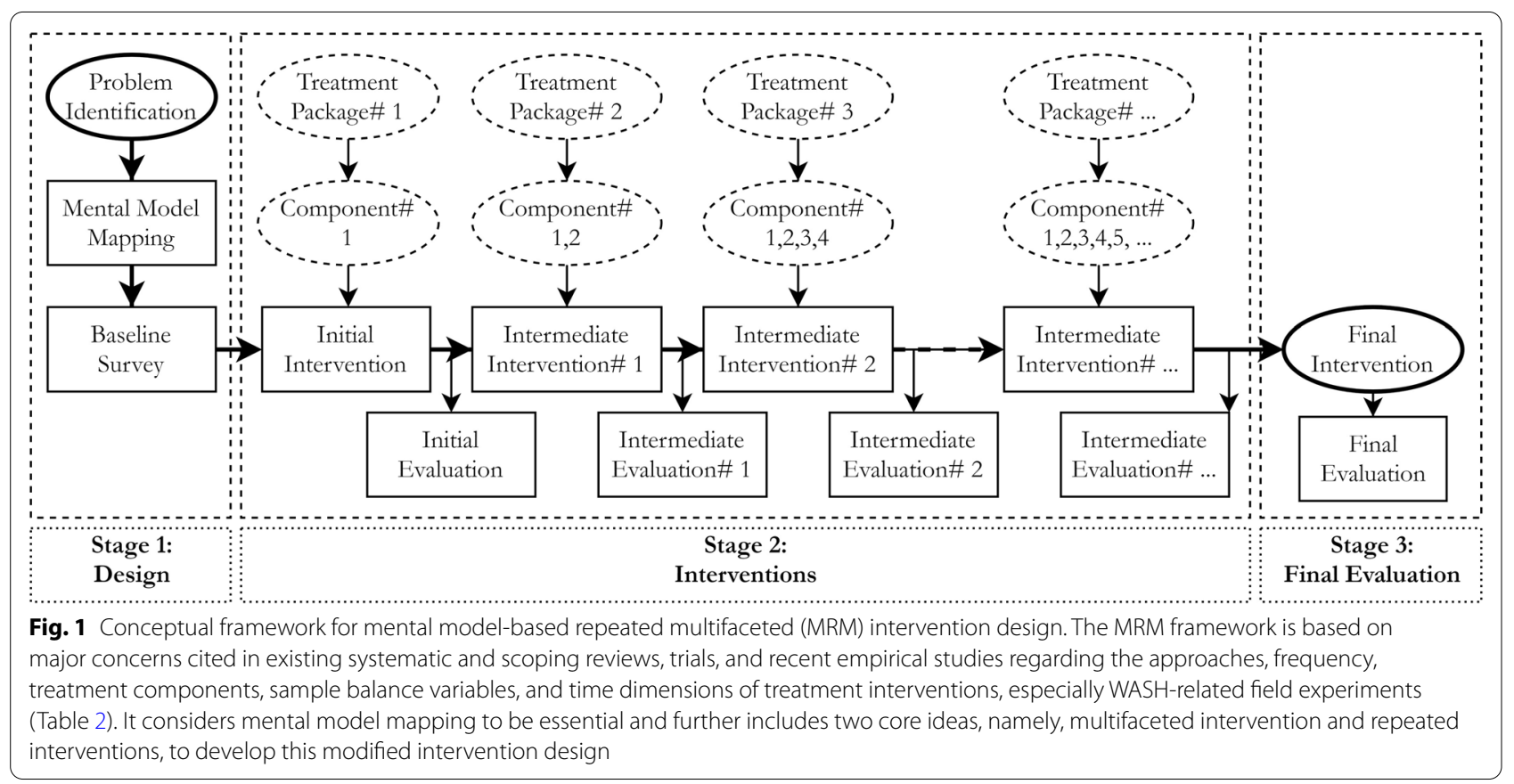

\section{Results}

\section{Concerns related to low or no intervention effects}

Based on our review of the literature and synthesis of the evidence, we found three major areas of concern in intervention design and implementation; these concerns underpin the theoretical foundation of our proposed framework. First, standard intervention design assumes that treatment subjects are similarly willing to adopt suggested preventive behaviors and have similar cognitive capacities; in fact, individuals have different cognitive capacities and mental models. These differences may lead to low-level outcomes. Human behavior is influenced by human attitudes, knowledge, perceptions, social norms, and beliefs $[10,21]$; these elements together help 
constitute individuals' mental models. Therefore, it is crucial to identify whether one of these elements could potentially affect intervention outcomes. Second, the impacts of single and short-term interventions wane over time $[7,19]$ because lasting behavioral change requires long-term intervention. Third, the responses of treatment groups to a specific intervention may vary under different or changing circumstances; most of the time, this concern has not been fully recognized. For example, a few individuals may prefer monetary incentives to behavioral nudging while unemployed. In addition, some treatment groups may respond better to behavioral nudging and hands-on experience than other groups [19, 22].

\section{Conceptual framework}

The MRM intervention design framework assumes mental model mapping to be essential, and it further includes two core ideas: multifaceted intervention and repeated interventions (Fig. 1). First, mapping mental models in the design stage allows the researcher or program analyst to understand a treatment group's mental or cognitive abilities with regards to adopting suggested preventive behaviors, as well as their willingness to do so (Stage 1 of Fig. 1). Sample balance tests need to consider mental model-related variables (e.g., flexibility), along with socioeconomic and demographic variables such as age, sex, education, income, and wealth. Mapping mental models before and after each intervention is particularly important, as changes can then be identified across time and treatments.

Second, multifaceted interventions allow multiplecomponent treatments and can generate longer-lasting effects than single-component interventions [23]. For example, different types of educational, financial, and behavioral interventions at different intensity levels (e.g., low, standard, high) can be combined to design a treatment package (Stage 2 of Fig. 1). However, the lengths and intensities of the treatments may differ, and they should be tailored to the relevant behavior-related outcomes. In some cases, both monetary and behavioral interventions are essential in encouraging the treatment groups to adopt a behavior. Third, repeated interventions may produce more pronounced intervention outcomes (Stage 2 of Fig. 1) and are more effective than single interventions. While the impact of a single intervention wanes over time, mixed interventions with multiple rounds are more likely to produce the anticipated outcomes.

\section{Discussion}

\section{Intervention design and implementation}

The MRM framework proposes a basic intervention design with three main features: mental model mapping, repeated interventions, and multifaceted interventions.
In Stage 1, mapping the mental models of a treatment group before or during the baseline survey is essential to designing the initial intervention (Fig. 1). A mental model is an overall representation of an individual's characteristics (e.g., attitudes, values, beliefs, social and cultural norms) that explains the individual's reasoning, inferencing, and decision-making processes. These processes influence the individual's ability to grasp, and willingness to accept suggested health behaviors [8, 9, 24-26]. Mapping mental models using modified versions of available methods $[8,26,27]$ would provide insights into an individual's or household responder's behavioral and cognitive capacity as they relate to the adoption of the suggested health behaviors.

Stage 2 includes two different types of interventions: initial and intermediate (Fig. 1). The number of intermediate interventions, as well as their type (e.g., informational, educational, financial, behavioral) and sequence (e.g., informational-practical-behavioral, informationalbehavioral-practical), should be adjusted in line with a program's short- and long-term goals. Researchers will need to identify the appropriate length (e.g., short-, medium-, long-term) and intensity (e.g., low, standard, high) of each treatment, depending on their research goals.

In the final evaluation (Stage 3), researchers will compare the final outcomes with the baseline and intermediate outcomes to arrive at conclusions regarding specific stage-level outcomes. Redesign will be necessary if the initial treatment produces lower-than-expected outcomes.

\section{Hypothetical intervention design}

A hypothetical repeated multifaceted intervention design is presented in Table 2. Each component has three distinct features, which are the intervention type, length, and intensity. A standard information component can be employed in the short-term in the initial stage. In the final stage, five different components can be employed sequentially as a treatment package. This sequence could be a cluster of mixed interventions in which the order of interventions (and their close variants) is based on the mental models of the treatment group members and expected outcomes from the programs. Thus, individuals with limited learning or adoption capacity, for instance, could be treated with higher intensity.

\section{Outcome analysis}

As each intervention combines multiple treatments, researchers should consider all the treatments at a given stage as a treatment package (e.g., treatments 1,2 , and 3 in combination are a treatment package for intermediate intervention \#2). Comparing the outcomes of each 
Table 2 An example of a repeated multifaceted intervention design

\begin{tabular}{|c|c|c|c|c|c|c|}
\hline \multirow[t]{2}{*}{ Round } & \multicolumn{6}{|c|}{ Intervention Component } \\
\hline & Feature & Comp.\#1 & Comp.\#2 & Comp. \#3 & Comp. \#4 & Comp. \#5 \\
\hline \multirow[t]{4}{*}{ Initial } & Type & Informational & & & & \\
\hline & Length & Short-term & & & & \\
\hline & Intensity & Standard & & & & \\
\hline & Evaluate & With baseline & & & & \\
\hline \multirow[t]{4}{*}{ Int. \#1 } & Type & Practical & Behavioral & & & \\
\hline & Length & Short-term & Medium-term & & & \\
\hline & Intensity & Standard & Low-level & & & \\
\hline & Evaluate & With baseline a & interventions & & & \\
\hline \multirow[t]{4}{*}{ Int. \#2 } & Type & Informational & Practical & Financial & Promotional & \\
\hline & Length & Short-term & Medium-term & Short-term & Short-term & \\
\hline & Intensity & Minimum & Low-level & Standard & High-level & \\
\hline & Evaluate & With baseline, i & intermediate \#1 & ions & & \\
\hline \multirow[t]{4}{*}{ Final } & Type & Informational & Practical & Behavioral & Financial & Promotional \\
\hline & Length & Short-term & Medium-term & Short-term & Short-term & Short-term \\
\hline & Intensity & Low-level & High-level & Low-level & Standard & Low-level \\
\hline & Evaluate & \multicolumn{5}{|c|}{ With baseline, initial, intermediate \#1, and intermediate \#2 interventions } \\
\hline
\end{tabular}

Int. Intermediate, Comp. Component

intervention with previous interventions (e.g., comparing intermediate intervention \#2 with the baseline and with intermediate intervention \#1) will be necessary to reveal whether the effects of a treatment package have persisted. If a promotional component is included as a treatment, the possibility of courtesy bias [1] on the part of the responders during after-intervention data collection should be accounted for to minimize the bias in outcome estimates.

Most importantly, researchers will need to check the mental model after each intervention to compare it with the initial mental model, the subject's willingness to accept the behavioral change (as stated in a baseline survey), and the subject's actual or demonstrated willingness to accept the suggested behaviors. Sub-group analysis is essential to assess the adherence to suggested behaviors by different groups within or between treatment groups. A crossover design would allow for various evaluation techniques, such as a quasi-experimental design (e.g., pre-post) for the initial intervention and an experimental design (e.g., difference-in-difference) for the intermediate and final interventions.

\section{Design, implementation, and analysis challenges}

First, individual or household responder-level mental models vary contextually; therefore, a suitable mental model mapping and classification procedure needs to be adopted that takes the prevailing experimental contexts into consideration. A professional behavioral profiler is needed to ensure accuracy, as typical enumerators are not trained to perform mental model mapping. Second, identification of the appropriate lengths and intensities of different treatments will be challenging during the initial and first intermediate stages due to various contextual factors. Researchers could use these two stages to test the initial treatments and identify appropriate treatment conditions to employ in the later stages. Third, the effect size of intermediate interventions may be misleading due to a variety of outside factors (e.g., unexpected bad or good weather).

\section{Conclusion}

Mental model mapping can reveal a treatment group's mental or cognitive abilities to adopt the suggested preventive behaviors during a multifaceted program intervention. Therefore, the inclusion of a mental modelrelated variable as a part of the balance test is critical to understanding the cognitive ability and willingnessrelated balances between different treatment and control groups. It is also important to consider the various types, lengths, frequencies, intensities, and sequences of treatments to design repeated multifaceted interventions and to consider mental model mapping with the intention of improving the program effectiveness of preventive health behaviors and outcome-related interventions.

\section{Limitations}

Our proposed framework is designed to provide an intervention model to improve health education and promotional intervention programs. An evaluation of actual 
effectiveness of field experiments using this framework is essential to examining our hypotheses and advancing our understanding of how MRM design can be applied to improve preventive health behaviors and outcomes.

\section{Abbreviations}

MRM: Mental model-based repeated multifaceted;WASH: Water, sanitation, and hygiene.

\section{Supplementary Information}

The online version contains supplementary material available at https://doi. org/10.1186/s13104-021-05516-9.

Additional file 1: Table S1. Literature search strategy for MRM intervention design, 2010-2020.

Additional file 2: Figure S1. Steps in identification of key concerns and framework development.

\section{Acknowledgements}

We would like to thank the conference participants of the Health Impact Assessment (organized jointly with the Environment section) session of the APHA's 2020 Virtual Annual Meeting and Expo, USA, and members of ResEcon for their helpful comments and suggestions. MGA acknowledges support from a postdoctoral travel grant from the University of Nebraska-Lincoln.

\section{Authors' contributions}

MGA conceived the original idea and developed the logical sequence of the intervention design flowchart, interpreted data from the overview of published articles, and wrote the first draft using literature jointly sourced by MGA and FT. Both authors read and approved the final manuscript.

\section{Funding}

None.

\section{Availability of data and materials}

Not applicable.

\section{Declarations}

Ethics approval and consent to participate

Not applicable.

\section{Consent for publication}

Not applicable.

\section{Competing interests}

The authors declare that they have no competing interests.

\section{Author details}

1 University of Nebraska-Lincoln, 140 Keim Hall, 1825 N 38th St, Lincoln, NE 68583, USA. ${ }^{2}$ Sylhet, Bangladesh.

Received: 24 November 2020 Accepted: 5 March 2021

Published online: 19 March 2021

\section{References}

1. Luby S, Rahman M, Arnold B, et al. Effects of water quality, sanitation, handwashing, and nutritional interventions on diarrhoea and child growth in rural Bangladesh: a cluster randomised controlled trial. Lancet Glob Heal. 2018;6:e302-15.

2. Null C, Stewart C, Pickering A, et al. Effects of water quality, sanitation, handwashing, and nutritional interventions on diarrhoea and child growth in rural Kenya: a cluster-randomised controlled trial. Lancet Glob Heal. 2018;6:e316-29.

3. Ahamad M. A note on behavioral aspects of health education and promotion interventions; 2019. https://papers.ssrn.com/abstract=3495680. Accessed 20 Feb 2021.

4. Humphrey J, Mbuya M, Ntozini R, et al. Independent and combined effects of improved water, sanitation, and hygiene, and improved complementary feeding, on child stunting and anaemia in rural Zimbabwe: a cluster-randomised trial. Lancet Glob Heal. 2019:7:132-79.

5. Fewtrell L, Kaufmann R, Kay D, et al. Water, sanitation, and hygiene interventions to reduce diarrhoea in less developed countries: a systematic review and meta-analysis. Lancet Infect Dis. 2005;5:42-52.

6. Gera T, Shah D, Sachdev H. Impact of water, sanitation and hygiene interventions on growth, non-diarrheal morbidity and mortality in children residing in low- and middle-income countries: a systematic review. Indian Pediatr. 2018;55:381-93.

7. White H, Raitzer D. Impact evaluation of development interventions: a practical guide. Manila: Asian Development Bank; 2017.

8. Datta S, Mullainathan S. Behavioral design: a new approach to development policy. Rev Income Wealth. 2014;60:7-35.

9. World Bank. Thinking with mental models. In: World Bank Group, editor. World development report 2015: mind, society, and behavior. Washington, DC: World Bank; 2015. p. 62-77.

10. Thaler R. Mental accounting matters. J Behav Decis Mak. 1999;12:183-206.

11. Johnson-Laird P. Mental models: towards a cognitive science of language, inference, and consciousness. 6th ed. Cambridge: Harvard University Press; 1983

12. Beaubien R, Parrish $\mathrm{S}$. The great mental models volume 1: general thinking concepts. Ottowa: Latticework Publishing Inc; 2019.

13. Dreibelbis $R$, Winch $P$, Leontsini $E$, et al. The integrated behavioural model for water, sanitation, and hygiene: a systematic review of behavioural models and a framework for designing and evaluating behaviour change interventions in infrastructure-restricted settings. BMC Public Health. 2013:13(1015):2-13.

14. Rychetnik L, Frommer M, Hawe P, et al. Criteria for evaluating evidence on public health interventions. J Epidemiol Community Health. 2002;56:119-27.

15. Hawe P. Lessons from complex interventions to improve health. Annu Rev Public Health. 2015:36:307-23.

16. Buchmann N, Field E, Glennerster R, et al. Throwing the baby out with the drinking water: unintended consequences of arsenic mitigation efforts in Bangladesh. Cambridge: Harvard University; 2019.

17. Mosler H-J. A systematic approach to behavior change interventions for the water and sanitation sector in developing countries: a conceptual model, a review, and a guideline. Int J Environ Health Res. 2012;22:431-49.

18. Michie $S$, West $R$, Sheals $K$, et al. Evaluating the effectiveness of behavior change techniques in health-related behavior: a scoping review of methods used. Transl Behav Med. 2018;8:212-24.

19. Ferraro P, Price M. Using nonpecuniary strategies to influence behavior: eevidence from a large-scale field experiment. Rev Econ Stat. 2013;95:64-73.

20. Craig P, Katikireddi S, Leyland A, et al. Natural experiments: an overview of methods, approaches, and contributions to public health intervention research. Annu Rev Public Health. 2017;38:39-56.

21. Michie S, van Stralen M, West R. The behaviour change wheel: A new method for characterising and designing behaviour change interventions. Implement Sci. 2011;6:42.

22. Cohen I, Fernandez Lynch H, Robertson C, editors. Nudging Health: Health Law and Behavioral Economics. Baltimore: Johns Hopkins University Press; 2016

23. Banerjee A, Duflo E, Goldberg N, et al. A multifaceted program causes lasting progress for the very poor: evidence from six countries. Science (80-). 2015;348:1260799-1260799.

24. Ahamad M. Mental model mapping for behavioral program design Behav Soc Sci Nat Res; 2019. https://socialsciences.nature.com/posts/ 48694-mental-model-mapping-for-behavioral-design. Accessed 1 July 2020.

25. Ajzen I. The theory of planned behavior. Organ Behav Hum Decis Process. 1991:50:179-211. 
26. Kelly $M$, Barker M. Why is changing health-related behaviour so difficult? Public Health. 2016;136:109-16.

27. Hoffman M, Lubell M, Hillis V. Linking knowledge and action through mental models of sustainable agriculture. Proc Natl Acad Sci.

2014;111:13016-21.

\section{Publisher's Note}

Springer Nature remains neutral with regard to jurisdictional claims in published maps and institutional affiliations.
Ready to submit your research? Choose BMC and benefit from:

- fast, convenient online submission

- thorough peer review by experienced researchers in your field

- rapid publication on acceptance

- support for research data, including large and complex data types

- gold Open Access which fosters wider collaboration and increased citations

- maximum visibility for your research: over 100M website views per year

At BMC, research is always in progress.

Learn more biomedcentral.com/submissions 